\title{
ADJUVANT STEREOTACTIC PERMANENT SEED BREAST IMPLANT: A BOOST SERIES IN VIEW OF PARTIAL BREAST IRRADIATION
}

\author{
Nicolas Jansen, M.D., Jean-Marie Deneufbourg, M.D., and PhilipPe Nickers, Ph.D. \\ Department of Radiation Oncology, Liege University Hospital, Liege, Belgium
}

\begin{abstract}
Purpose: The aim of this study was to use permanent seed implants in the breast and describe our experience with 15 cases, using iodine seed implants as a tumor bed boost.

Methods and Materials: Breasts were fixed with a thermoplastic sheet, a template bridge applied, the thorax scanned and the images rotated to be perpendicular to the implant axis. Skin, heart, and lung were delineated. A preplan was made, prescribing 50 Gy to the clinical target volume (CTV), consisting in this boost series of nearly a quadrant. Iodine (125) seeds were stereotactically implanted through the template, and results were checked with a postplan computed tomographic (CT) scan.

Results: The breast was immobilized reproducibly. Simulation, scanning, and implant were performed without difficulties. Preplan CTV D90\% (the dose delivered to $90 \%$ of the CTV) was $66 \mathrm{~Gy}$, and postoperative fluoroscopic or CT scan checks were satisfactory. Pre- and postplan dose-volume histogram showed good organ sparing: mean postplan skin, heart, and lung $\mathrm{V} 30 \mathrm{~Gy}$ (the organ volume receiving a dose of $30 \mathrm{~Gy}$ ) of $2 \pm 2.2$ $\mathrm{mL}, 0.24 \pm 0.34 \mathrm{~mL}$, and $3.5 \pm 5 \mathrm{~mL}$, respectively. No short-term toxicity above Grade 1 was noted, except for transient Grade 3 neuropathy in 1 patient.

Conclusions: Seeds remained in the right place, as assessed by fluoroscopy, absence of significant pre- to postplan dose-volume histogram change for critical organs, and total irradiated breast volume. The method could be proposed as a boost when high dosimetric selectivity is required (young patients after cardiotoxic chemotherapy for left-sided cancer). This boost series was a preliminary step before testing partial breast irradiation by permanent seed implant in a prospective trial. () 2007 Elsevier Inc.
\end{abstract}

Permanent seed implant, Breast boost, Partial breast irradiation, Stereotactic brachytherapy.

\section{INTRODUCTION}

Breast-conserving treatment for early breast cancer involves surgery and adjuvant radiotherapy of the whole breast and often a boost to the tumor bed. The results of this classical treatment are good, with local control rates of more than $95 \%$ at 5 years and good cosmesis $(1,2)$. As early breast cancer treated by adequate surgery has few local recurrences outside the quadrant of the initial tumor (3), a partial breast irradiation of the area of the initial location with a margin can be applied. For selected low-risk patients, this approach has been proposed using interstitial brachytherapy, external beam irradiation, or intraoperative irradiation with a mobile linear accelerator $(4,5)$. The suggested advantages of these approaches include the precise treatment of the target volume, the avoidance of a 5-6-week treatment series, less skin toxicity, and lower cardiac as well as pulmonary doses. If patients are carefully selected and rigorous quality control measures are applied, the 7-year local control rates and cosmetic outcome data obtained seem to be at least as good as those obtained after the classic treatments $(4,5)$. Randomized comparisons between the whole breast and the partial breast irradiation approach are, however, still ongoing (Communicated during The GECESTRO [Groupe Européen de Curie Therapie-European Society for Therapeutic Radiology and Oncology] Premeeting workshop on brachytherapy for breast cancer, May 5, 2005, Budapest, Hungary, afternoon session on accelerated partial breast irradiation-future directions.)

Arguments against partial breast irradiation include the present difficulty in accurately defining the clinical target volume (CTV) and the possible migration of markers or of any other material that is placed in the tumor bed, such as seeds (6). Using experience gained from prostate implants, we developed a permanent seed implantation technique for the breast based on a stereotactic approach-"stereotactic" being defined as "a technique for surgical treatment or scientific investigation that permits the accurate positioning of probes inside ... the body, based on three-dimensional diagrams" (7). Although the value of this implant for partial
Reprint requests to: Nicolas Jansen, M.D., Liege University Hospital, Domaine Universitaire Sart Tilman B35, B-4000 Liege, Belgium. Tel: (+32) 43667462; Fax: $(+32)$ 43667952; E-mail: nicolas.jansen@chu.ulg.ac.be
Conflict of interest: A grant was received from IBt, which also provided the implanted seeds.

Received July 1, 2006, and in revised form Sept 22, 2006. Accepted for publication Oct 9, 2006. 


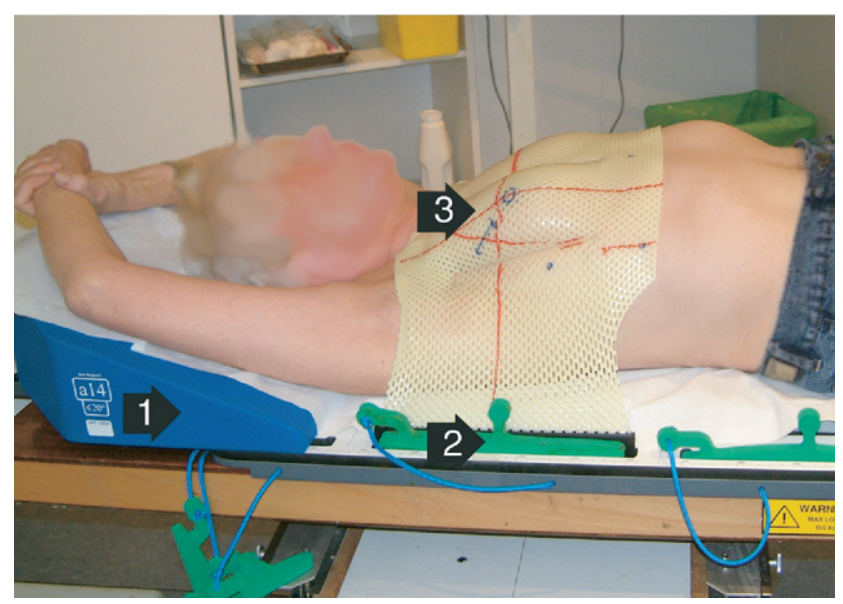

Fig. 1. Breast immobilization with a thermoplastic sheet. The patient is placed on a base plate and arms are positioned on a blue armrest (1). The yellow thermoplastic sheet is fixed to the base plate with green table inserts (2). The needle-guiding template has been removed to show the blue marking points on the thermoplastic sheet, such as nipple, scar, and tattoo points (3).

breast irradiation will be tested in a future phase II study, we proposed this particularly selective brachytherapy technique (which is currently used in prostate cancer) to patients as an alternative to a classical interstitial iridium brachytherapy boost.

\section{METHODS AND MATERIALS}

\section{Patient selection}

After external beam adjuvant whole-breast radiotherapy, a boost was proposed owing to the presence of one or more risk factors that are possibly associated with local relapse. These factors included the patient being less than 50 years of age, resection margins that were considered to be insufficient (unknown or positive margins or margins of less than $2 \mathrm{~mm}$ ), and Grade 3 lesions. Patients with T3-T4 lesions, very small or very large breasts, a very eccentric tumor bed localization, or a history of breast reconstruction were excluded. Patients either not able to have a computed tomographic (CT) or magnetic resonance imaging (MRI) scan (due to, for example, claustrophobia) or those who refused to have such a scan, as well as those who were not capable of understanding basic radioprotection rules, or who were involved in the daily care of young children, were not entered. We treated patients with or without the presence of surgical clips placed during initial surgery to help identify the target volume. No chemotherapy was ongoing during the external beam radiotherapy or planned for afterwards. Patients were offered this permanent implant boost method, which is now well established in numerous anatomic regions of the body, as an alternative to the classical iridium boost. The differences explained included the greater dosimetric selectivity and the radioprotection rules. Patients were clearly informed about the technique and agreed to the treatment.

\section{External beam radiotherapy}

Patients underwent classical external beam adjuvant wholebreast radiotherapy to a dose of 46-50 Gy in 23-25 fractions using two opposed tangential, wedged, nonisocentric beams with a 6-megavolt linear accelerator or a cobalt ${ }^{60}$ machine. Treatments were given over a 5-6-week period. The dose was reported according to International Commission on Radiation Units and Measurements guidelines (8).

\section{Brachytherapy simulation}

Simulation was performed using the "all in 1" (AIO) immobilization system (Orfit Industries, Wommelgem, Belgium), which consisted of an indexed base plate on the table; head-and-neck supports; and arm, knee, back, and ankle cushions. The patient was placed in a supine position, with both arms behind the head (Fig. 1). Under fluoroscopic control, the isocenter was placed in the middle of the tumor bed, as indicated by radiopaque surgical clips. In the absence of these markers, the tumor bed was identified based on clinical examination, ultrasound and mammography data. Without changing the isocenter, the gantry was turned to check at which angle the tumor bed did not project onto the lungs or the thoracic wall. This gantry angle defined the directional axis for the later needle implant. A heated, thick, perforated thermoplastic sheet of $35 \mathrm{~cm} \times 45 \mathrm{~cm}$ (Orfit Industries, Wommelgem, Belgium) was placed on the thorax, molded around both breasts, and fixed to the immobilization plate using small inserts fixed to the sheet (Fig. 1).

We developed a new "template bridge" consisting of a parallel medial and a lateral perforated template, each with a thickness of $1.7 \mathrm{~cm}$ and linked by a slidable arm (Fig. 2). This was applied on the thermoplastic sheet at the level of the breast, slightly compressing it during the cooling phase. The templates were placed so that, as far as possible, they were perpendicular to the directional axis. The isocenter was once more checked, and a set of orthogonal reference radiographs were obtained. Six tattoo points were available from previous external beam radiotherapy ( 3 medial and 3

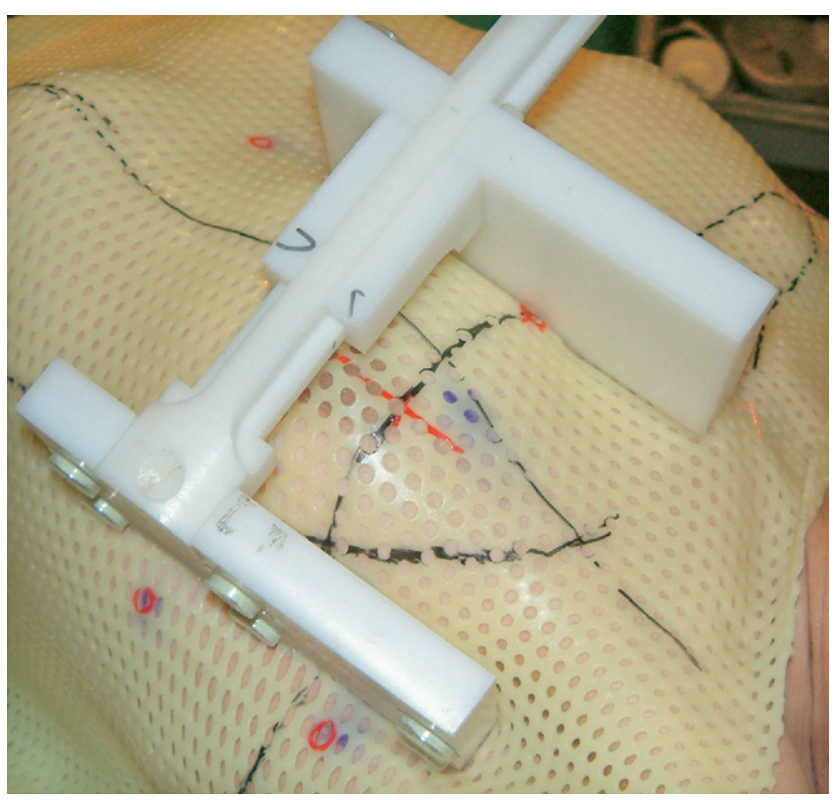

Fig. 2. Needle-guiding template bridge. A clear, yellow thermoplastic sheet is molded around the breast. A slidable, white template bridge mildly compresses the breast. Black lines indicate the axes and the isocenter. The red line and dots mark, respectively, a scar and tattoo points. Markers that are compatible with magnetic resonance imaging are present on the outer surface of the templates. 

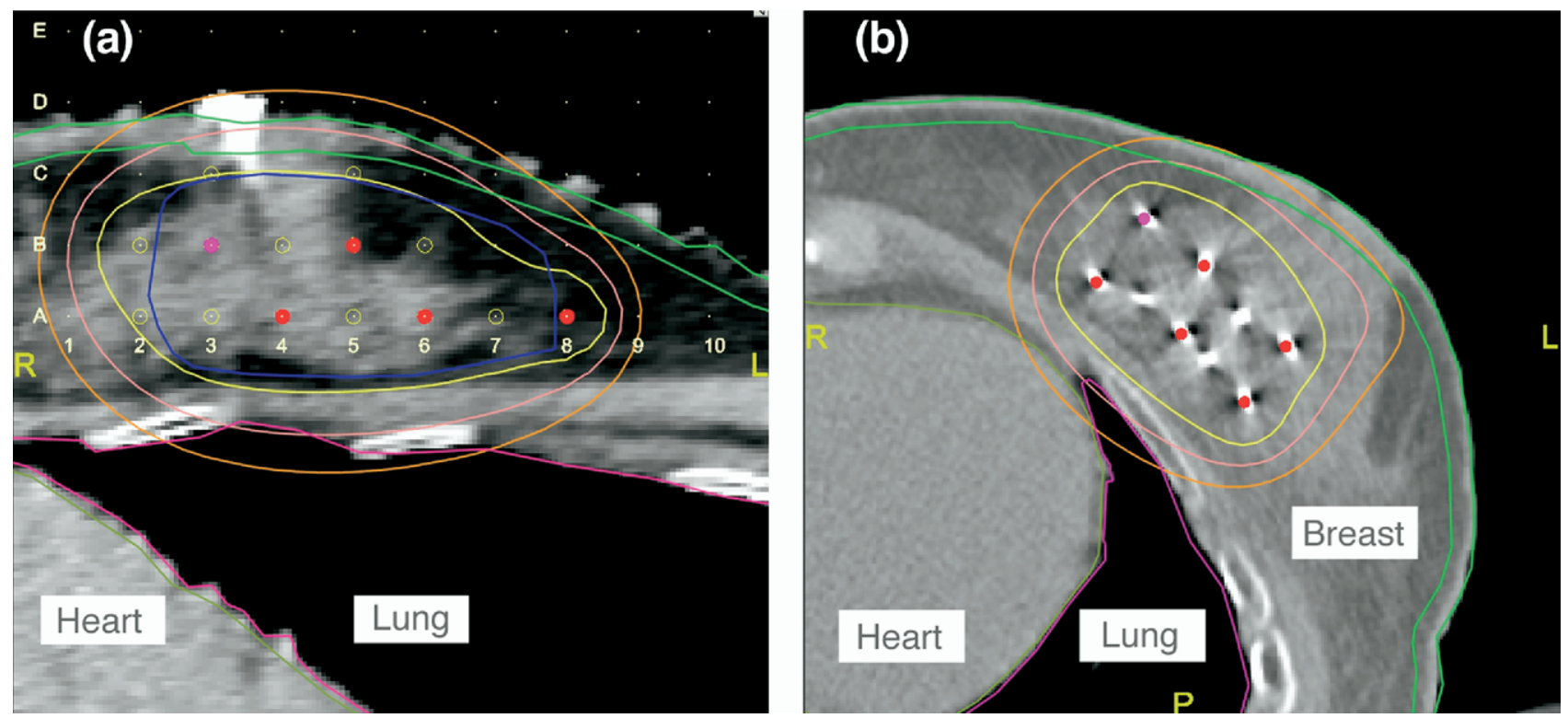

Fig. 3. (a) Preplan dosimetry. The skin is indicated in light green, the lung in pink, and the heart in dark green. An opaque marker that is placed on the nipple is visible in white. The template positions are shown by the small, regularly placed white dots: the columns are marked 1 to 10 and the lines A to E. The large red dots indicate source positions in this plane. Open yellow dots indicate the presence of seeds in other planes. The target volume is indicated in blue. The yellow line indicates the prescription isodose of $50 \mathrm{~Gy}$. The soft pink line indicates the $30 \mathrm{~Gy}$ isodose line; the light orange line indicates the $20 \mathrm{~Gy}$ isodose line. (b) Postplan dosimetry. The skin is indicated in light green, the lung in pink, and the heart in dark green. The large red dots indicate source positions in this plane. The yellow line indicates the prescription isodose of $50 \mathrm{~Gy}$. The soft pink line indicates the $30 \mathrm{~Gy}$ isodose line; the light orange line indicates the 20 Gy isodose line. The good protection of skin, heart, ant lung can be noted.

lateral, at the base of the breast, equally spread in the cranio-caudal direction). These tattoo points were the main reference points that were marked on the thermoplastic sheet. Other points included nipple position and scars. These references enabled the thermoplastic sheet to be replaced, during subsequent stages, in exactly the same position on the breast and on the base plate, thus offering a reproducible immobilization. The fixed head support and arm cushion also forced the patient to remain in the same position. During simulation, the breast was photographed to document the procedure. The cosmetic state of the breast was evaluated by both patient and physician, using the categories "excellent", "good", "reasonable", and "bad".

\section{Imaging}

Magnetic resonance imaging/CT-compatible markers were placed on the corners of the external surface of the medial and lateral templates and on the nipple. The sheet was repositioned exactly using the reference and tattoo points for guidance. A set of $\mathrm{T} 1$ and T2 images (MRI) or CT images were obtained, scanning the whole ipsilateral thoracic area, the heart, and the whole template bridge. Slices were $3 \mathrm{~mm}$ thick and were obtained every 3 $\mathrm{mm}$. The images were then sent to the treatment planning system workstation.

\section{Target volume definition and preplanning}

The image set was first imported into a custom-made application known as Slicer (Socobel, Waterloo, Belgium), which reconstructed planes perpendicular to the directional axis, thus parallel to the templates. These reconstructed images were then sent to the Prowess brachytherapy treatment planning system (v4.2; Prowess
Inc., Chico, CA). A virtual template was applied, with a distance between the needle rows and columns of $10 \mathrm{~mm}$, which corresponded to the holes that were drilled in the templates. For this series of patients, the implant direction selected was from lateral to medial. The CTV was defined as the tumor bed, as indicated by clips, seroma, and scar tissue plus a margin of $20 \mathrm{~mm}$, without going into skin or thoracic cage, but extending to the retro-areolar area-this amounted, in practice, to a breast quadrant. Additional information used for setting the CTV location, certainly in the absence of markers in some patients, was the preoperative set of mammographies, a postoperative breast ultrasound performed for this purpose, clinical data from the patient file, and the pathologic report. Skin was defined as a 4-5-mm-wide structure, taking into account that skin toxicity can depend on doses to skin vasculature that can be found in this range (9). Organs at risk (skin, lung, and heart) and CTV were delineated on the reconstructed slices. Virtual iodine-125 sources, at a minimum distance of $10 \mathrm{~mm}$ from each other, were then applied in a staggered manner. They were placed in and around the CTV, avoiding sources close to the skin or the thoracic wall (Fig. 3a). Only strands were allowed.

\section{Prescription dose}

The time-dose factor (TDF) of a "classical" external beam boost of $16 \mathrm{~Gy}$ in 8 fractions of $2 \mathrm{~Gy}$ at 5 fractions per week is 26 (10). For a permanent seed implant, the total dose $D$ in cGy and TDF are related by Eq. 1, where $\lambda$ is the decay constant of the isotope $(10,11)$.

$$
D=\left(\frac{T D F}{3.53 \times 10^{-3} \times \lambda^{0.35}}\right)^{0.7407}
$$


In this case, the decay constant $\lambda$ for iodine-125 is defined by Eq. 2 , with the $t_{1 / 2}$ (half-life) of iodine-125 being $1,425.6 \mathrm{~h}$.

$$
\lambda=\left(\frac{\ln (2)}{t_{1 / 2}}\right) h^{-1}=4.8621 \times 10^{-4}
$$

From Eqs. 1 and 2, the calculated theoretical dose is 52.9 Gy. Taking into account the given dose by external radiotherapy, and based on our prostate experience, we selected 50 Gy given at a very low dose rate as the prescription dose (PD) for this boost treatment. In previously published work on permanent breast seed implants, a similar approach was used (12). The $50 \mathrm{~Gy}$ isodose line should cover the CTV on the preplan with, for as far as possible, a margin of $5 \mathrm{~mm}$ to compensate for the possible seed shift during the implantation procedure. D90 was the dose delivered to $90 \%$ of the CTV. CTV V50 Gy (volume receiving $50 \mathrm{~Gy}$ ) and CTV V100 Gy were the CTV volumes receiving $100 \%$ or $200 \%$ of the prescribed dose. For skin, heart, and lung, the V5 Gy, V15 Gy, and V30 Gy were the organ volumes receiving doses of 5 Gy, 15 Gy, and $30 \mathrm{~Gy}$. We tried to avoid giving more than $30 \mathrm{~Gy}$ to the skin, accepting however local hotspots. This skin dose level corresponds to two-thirds of the prescribed dose, which is similar to our experience in high-dose-rate breast boosting. Finally, the total volume of breast receiving $100 \%$ and $200 \%$ of the PD was defined as Total Breast V50 Gy and V100 Gy. Volumes irradiated were reported in milliliter. All these preplan dose-volume histogram data were noted, and an implant report was printed for use in the operating theater.

\section{Patient preparation}

The whole procedure was carried out at the one-day clinic. The patients were placed on the standardized "all in 1 " system in the simulated position and brought under general anesthesia. The thermoplastic sheet was disinfected by spraying it with an iodine solution (Isobetadine) and then placed onto the patient. The breast itself was repositioned inside the sheet using previously marked reference points (tattoos, scars, and the nipple position). Holes cut out in the thermoplastic sheet permitted final adjustments to be made to the breast position by the insertion of fingers. These holes also allowed needles to pass through the thermoplastic sheet. The gas-sterilized slidable bridge was then applied on the outside of the thermoplastic sheet in the imprint that was made during initial simulation. The distance between the templates was carefully checked. A single dose of corticosteroids (methylprednisolone $125 \mathrm{mg}$ ) was given to prevent excessive breast swelling.

\section{Needle implantation procedure}

First, one or more stabilization needles were placed in the upper plane(s) to avoid displacement of the breast. Following the implant scheme, needles were inserted, such as is done during prostate brachytherapy. They traveled through the breast and came out on the other side, guided by the templates. If the rigid needle easily appeared on the other side of the breast at the corresponding template hole, it was concluded that the trajectory was satisfactory. To avoid damaging the tips of the implant needles by breast tissue, skin, or templates, we first inserted a rigid needle with a trocar that had a pyramidal tip. The trocar was then retracted, leaving behind the needle cannula that linked both templates. A fine, long guidance trocar, without any extremity button, was then inserted into the cannula. The initial cannula was retracted and the definitive implant needle was carefully pushed over the long, fine guidance trocar, which was then retracted and replaced by the implant needle's own trocar. Source insertion was only started after all needles had been inserted.

\section{Source insertion}

Iodine-125 strands consisting of $10 \pm 0.300$ milliCurie seeds (IBt, Seneffe, Belgium) were cut to the correct length and seeds were calibrated just before the implant procedure. Needle by needle, the trocar was removed, and a source transfer container was attached. The strand was inserted and pushed by a thick trocar that was adapted to the transfer container. The needle was retracted to the correct insertion depth from the external surface of the lateral template, as calculated during preplanning, and the seeds were inserted as is done for prostate implants. Before and after each strand insertion, a rapidly obtained fluoroscopic image was inspected on the screen to confirm correct placement. At the end of the procedure, the template bridge was removed and seed placement was again checked under fluoroscopy. This was repeated after removal of the thermoplastic sheet, with the breast in a relaxed position (Fig. 4). The breast was gently cleaned, and general anesthesia was ended. The patient was then asked to wear, as often as possible, a solid sports bra to reduce breast movements.

\section{Radioprotection}

The same precautions were taken and the same material used as for prostate implants. The patient was instructed to avoid close and prolonged personal contact with others for the next 3 months.

\section{Postimplant plan}

On Day 14, a postplan CT of the breast with a sports bra was made. The patient was placed in a supine position. The same CT parameters were used as for the preplan CT. The same structures were delineated except for CTV (Fig. 3b). Total Breast V50 Gy

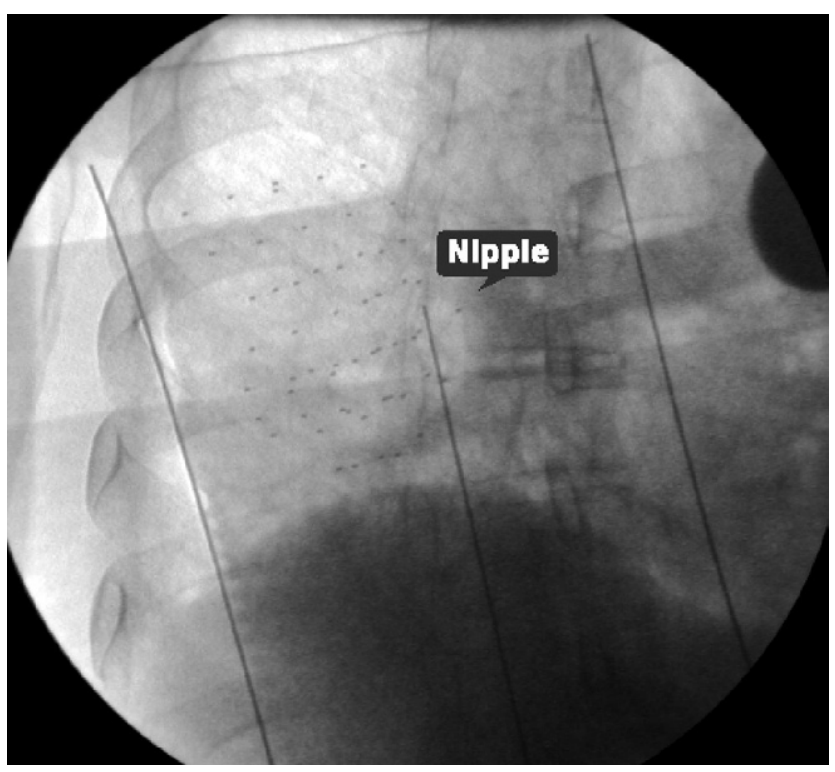

Fig. 4. Postimplant fluoroscopic image obtained after removal of the thermoplastic sheet showing the implanted strands. The two lateral opaque lines indicate the limits of the breast. The central opaque marker tip indicates the nipple location. The tumor was located between the upper and lower outer quadrant of the right breast. 
and V100 Gy were calculated, as well as the different doses for skin, lung, and heart. The statistical comparisons between preplan and postplan data were performed using a paired bilateral Student $t$ test.

\section{Follow-up}

Each patient was seen 2-3 weeks and 3 months after the implant procedure, and their progress was followed according to existing local guidelines. Cosmetic evaluation was planned from a 1-year follow-up onwards.

\section{RESULTS}

\section{Patient, tumor, and breast characteristics}

One of 3 patients to whom the technique was proposed refused this treatment modality, mainly due to concerns regarding their ability to respect radioprotection guidelines. From March to December 2005, 15 female patients were treated. The median age was 55 years (range, $41-74$ years). Five patients had a left-sided and 10 patients had a rightsided breast tumor. Three patients were premenopausal at the time of implant.

Most tumors were early: $13 \mathrm{~T} 1$ and $2 \mathrm{~T} 2$ lesions (TNM classification, International Union Against Cancer [UICC], 6th edition, 2002). The median maximal diameter was 13 $\mathrm{mm}$ (range, 6-37 $\mathrm{mm}$ ). Ten patients were node-negative and 5 had axillary N1 involvement. Three patients had an invasive lobular carcinoma, the others had an invasive ductal carcinoma. No patient had distal metastases. Evaluation by both patient and physician showed that all breasts were in an "excellent" or "good" cosmetic state before the implant.

\section{Preplanning data}

The median number of needles used was 14 (range, $8-20$ ); the median number of seeds per patient was 52 (range, 29-80). The mean preplan CTV volume was $58 \pm$ $23.2 \mathrm{~mL}$. For a PD of $50 \mathrm{~Gy}$, we observed a mean CTV D90 of $66 \pm 14.9 \mathrm{~Gy}$, a mean CTV V50 Gy of $97 \pm 3.9 \%$, and a mean skin V30 Gy of $6 \pm 3.9 \mathrm{~mL}$. Heart data were based on the 5 patients with a left-sided tumor. For every patient, the heart V30 Gy was zero $\mathrm{mL}$. The mean lung V30 Gy was 1.4 $\pm 2.5 \mathrm{~mL}$. The mean total breast V50 Gy and V100 Gy were $105 \pm 41.4 \mathrm{~mL}$ and $37 \pm 20 \mathrm{~mL}$, respectively (Table 1 ).

\section{Implant procedure}

The median time between external radiotherapy and breast implant was 13 days (range, 3-19 days). The median duration of general anesthesia was $150 \mathrm{~min}$. The fluoroscopic images that were obtained at the end of the implant procedure with and without the thermoplastic sheet were compared (Fig. 4). Seeds remained in place, notably within the planned breast quadrant, except for three strands that were not optimally placed owing to technical difficulties such as bleeding inside the implant needle.
Table 1. Dose-volume histogram data for a prescribed dose of 50 Gy (VLDR) $(n=15)$

\begin{tabular}{|c|c|c|c|c|c|}
\hline & \multicolumn{2}{|c|}{ Preplan } & \multicolumn{2}{|c|}{ Postplan } & \multirow[b]{2}{*}{$p^{*}$} \\
\hline & mean & $\pm \mathrm{SD}$ & mean & $\pm \mathrm{SD}$ & \\
\hline \multicolumn{6}{|l|}{ CTV } \\
\hline Volume & $58 \mathrm{~mL}$ & 23.2 & & & \\
\hline D90 & $66 \mathrm{~Gy}$ & 14.9 & & & \\
\hline V50 Gy & $97 \%$ & 3.9 & & & \\
\hline V75 Gy & $78 \%$ & 11.4 & & & \\
\hline V100 Gy & $50 \%$ & 19.3 & & & \\
\hline \multicolumn{6}{|l|}{ Skin } \\
\hline V5 Gy & $50 \mathrm{~mL}$ & 11.6 & $53 \mathrm{~mL}$ & 10.1 & 0.102 \\
\hline V30 Gy & $6 \mathrm{~mL}$ & 3.9 & $2 \mathrm{~mL}$ & 2.2 & 0.003 \\
\hline V50 Gy & $0.43 \mathrm{~mL}$ & 1.4 & $0.09 \mathrm{~mL}$ & 0.12 & 0.375 \\
\hline \multicolumn{6}{|l|}{ Heart $^{\dagger}$} \\
\hline V15 Gy & $0.06 \mathrm{~mL}$ & 0.13 & $4.7 \mathrm{~mL}$ & 5.6 & 0.133 \\
\hline V30 Gy & $0.0 \mathrm{~mL}$ & 0 & $0.24 \mathrm{~mL}$ & 0.34 & 0.186 \\
\hline \multicolumn{6}{|l|}{ Lung } \\
\hline V15 Gy & $15 \mathrm{~mL}$ & 13.6 & $22 \mathrm{~mL}$ & 19.9 & 0.145 \\
\hline V30 Gy & $1.4 \mathrm{~mL}$ & 2.5 & $3.5 \mathrm{~mL}$ & 5.0 & 0.104 \\
\hline \multicolumn{6}{|l|}{$\begin{array}{c}\text { Total breast } \\
\text { volume }\end{array}$} \\
\hline V50 Gy & $105 \mathrm{~mL}$ & 41.4 & $96 \mathrm{~mL}$ & 37.1 & 0.007 \\
\hline V100 Gy & $37 \mathrm{~mL}$ & 20 & $34 \mathrm{~mL}$ & 16.4 & 0.458 \\
\hline
\end{tabular}

Abbreviations: CTV = clinical target volume; Gy = Gray; $\mathrm{VLDR}=$ very low dose rate; $\mathrm{V}=$ volume; V50 Gy = volume in $\mathrm{mL}$ receiving a dose of $50 \mathrm{~Gy}$; $\mathrm{D} 90=$ dose in Gray received by $90 \%$ of a volume; SD $=$ standard deviation.

* Paired bilateral Student $t$ test.

${ }^{\dagger}$ Heart data are based on 5 patients with a left-sided tumor.

\section{Postimplant plan}

Mean skin V30 Gy was $2 \pm 2.2 \mathrm{~mL}$. Mean skin V50 Gy was below $0.1 \mathrm{~mL}$. We observed hotspots, the mean maximal skin dose in 1 point being $65 \pm 35 \mathrm{~Gy}$. Mean heart V30 Gy was $0.24 \pm 0.34 \mathrm{~mL}$, and mean lung V30 Gy was $3.5 \pm$ $5 \mathrm{~mL}$. Mean total breast V50 Gy decreased from $105 \pm 41$ $\mathrm{mL}$ in preplans to $96 \pm 37 \mathrm{~mL}$ in postplans $(p=0.007)$. Mean total breast V100 Gy did not change significantly (Table 1).

\section{Short-term follow-up data}

At the time of the postimplant CT scan, little toxicity was noted that could not be attributed to the previous external beam radiotherapy or the brachytherapy procedure. It consisted of Grade 1 skin toxicity, mild breast edema, and tenderness. The small scars from the individual needles were still visible at that time. One patient had a hematoma, and 1 patient complained of persisting fatigue. One patient developed a transient, totally reversible Grade 3 bilateral upper-limb neuropathy. At a mean follow-up of $10 \pm 2.5$ months, no other apparent treatment-related toxicity or local relapses were noted.

\section{DISCUSSION}

We have described a new technique for the permanent implantation of seeds in the breast. Imaging and precisely 
inserting seeds in a soft organ such as the breast does require new tools. We developed an adapted breast immobilization system, a slidable needle-guiding template bridge, a specific simulation procedure, a new image rotating application, and a preplan-based implant technique for the breast.

In our experience, it can be difficult to appreciate the relationship between needles and CTV using a reference needle under ultrasound control (12). Therefore, we devised a stereotactic system, based on immobilizing the breast with a thermoplastic sheet that can be precisely repositioned using a set of reference points on the sheet and on the patient's skin. Compared with other partial breast irradiation techniques using iridium-192, the whole brachytherapy procedure can be carried out in a 1-day clinic after simulation and scanning on an outpatient basis.

In general, seed deposits looked correct as indicated by postoperative fluoroscopic checks, except for three strands that had not been optimally placed because of technical difficulties such as bleeding inside the implant needle. Should the fluoroscopic evaluation after each strand insertion show a suboptimal placement, the problem can still be corrected intraoperatively, by adding extra seeds to the areas missed.

In this analysis, which aimed to cover a whole quadrant while sparing critical organs, no clinically relevant shift of seeds toward the skin or thoracic wall was observed as skin, heart, and lung volume histogram data did not change significantly from pre- to postplan; the only exception was for skin V30 Gy (Table 1). Total breast V50 Gy decreased slightly on postplans ( $p=0.007$ ), with a mean difference between the preand postimplant of $9.21 \pm 11.2 \mathrm{~mL}$ (confidence interval, 3.55-14.9). Meanwhile the total breast V100 Gy did not change significantly. We thus assume that the mild seed shift has a limited dosimetric impact on the periphery of the implant, rather then in the center of the implant where the hyperdosage areas are to be found. Should we want to account for this phenomenon, the security margin taken around the CTV on the preplan can be enlarged. As the initial tissue repair occurs normally within 2 weeks, we do not expect strands to move after the postplan evaluation. We conclude that, as with permanent prostate implants, postplan analysis shows a slight but acceptable degradation of the ideal plans that were designed preoperatively.

The apparently high mean preplan Total Breast V50 Gy $(105 \mathrm{~mL})$, in comparison with the mean CTV volume (58 $\mathrm{mL}$ ), can be explained by our loading method, which aims to surround the whole CTV to compensate for possible seed shifts as discussed above. Taking a 5-mm margin in all directions around a spherical volume of $58 \mathrm{~mL}$ leads more or less to a $105-\mathrm{mL}$ volume. However, the mean postplan V50 Gy value remains largely below $150 \mathrm{~mL}$, which is the cutoff value for the appearance of late side effects in a previous breast brachytherapy series (13).

We observed no adverse reactions or other important side effects except for a transient bilateral Grade 3 neurologic toxicity in 1 patient. We have, at present, no clear explanation for this temporary problem, but we suggest that it might have been related to the extension of both arms or to neck manipulation during the implant procedure. The short follow-up does not permit us to come to any conclusions about tumor control or cosmetic outcome.

The prescription dose of 50 Gy (very low dose rate) was calculated on the basis of previously published data using the TDF approach (10-12). We also recalculated the biologically equivalent dose (BED) of this treatment, taking into account repair and repopulation of breast tissues $(14,15)$, and obtained with an alpha/beta ratio of $10 \mathrm{~Gy}$ for early and tumor effects a BED in $\mathrm{Gy}_{10}$ that is similar to the classical 16 Gy boost in eight fractions. The BED in $\mathrm{Gy}_{3}$ for late effects in healthy tissues seems, on the other hand, to be higher than for the classical external approach. This is mainly the case for the high dose areas inside the CTV; the organs at risk around the CTV (skin and subcutaneous tissue, heart, and lung) receive a much lower dose because of the rapid dose fall-off and the dosimetric selectivity of this treatment, translating into a low BED. The mean postplan skin V30 Gy ( $=60 \%$ of the prescription dose) is, for instance, only $2 \mathrm{~mL}$, corresponding to a BED of $26 \mathrm{~Gy}_{3}$ at this point, which is equivalent to the classical treatment. It remains to be seen if these differences will lead to a different local late toxicity profile.

Target definition remains a challenging point in breast brachytherapy. In the present series, we wanted to treat a large boost volume (a breast quadrant) to a relatively low boost dose (16). This explains why, contrary to what is proposed in the Groupe Européen de Curiethérapie-European Society for Therapeutic Radiology and Oncology (GEC-ESTRO) randomized partial breast irradiation protocol, the margin is not adapted in function of the surgical margins that have already been taken, and "high-risk" patients were included. The absence of markers in some patients or the difficulty to distinguish seeds from markers of the tumor bed did contribute to the complexity of postplan analysis. Moreover, the breast position on postplans was slightly different because there was no compression by the thermoplastic sheet. Furthermore, the scan orientation is different as axial slides are obtained for the postplan, in contrast to the preplan reconstructed slides that are perpendicular to the directional axis. It is, of course, possible to obtain a postplan image series with the thermoplastic sheet in place, but this does not constitute a real treatment situation, even with patients continuously wearing a sports bra to reduce breast movements. Finally, most of the previously described breast brachytherapy treatments using plastic tubes, from which good results were reported, were also done on organs that do not remain immobile for the duration of the whole treatment (2). For all these reasons, we decided not to indicate CTV structures on postplans. We realize that, in future, we should be able to more precisely identify the postplan CTV and we are currently working on new ways to define, even before surgery, its future borders.

Because the breast is more exposed than the prostate area, there is a higher dose rate at $1 \mathrm{~m}$ from the patient. For the boost technique described with a low PD and using iodine125 seeds, measurements after implantation did, however, 
not show a dose rate beyond safe or legal limits (17). A higher PD for exclusive breast treatments might require a larger number of seeds or a higher seed activity, or both. This could increase the dose rate at $1 \mathrm{~m}$ in air beyond acceptable limits, unless palladium-103 seeds rather than iodine-125 seeds are used (18).

Dose inhomogeneity is inherent to all brachytherapy treatments, and particularly in the center of larger implants continuous high dose areas tend to be present. Should we want to avoid this, it is possible to use a different loading pattern or two or more different seed activities, placing the lower activity strands in the center of the implant.

This analysis was considered to be a necessary preliminary step before starting with partial breast irradiation with permanent implants in a prospective trial. We think, however, that the present method could also be proposed as a boost in cases in which a high dosimetric selectivity might be required, such as when young patients with left-sided breast cancer have previously been treated with cardiotoxic chemotherapy. Reducing heart doses can be expected to decrease long-term cardiac toxicity (19). Preimplant and postimplant dose-volume histogram data do, indeed, show low heart, lung, and skin doses. Moreover, all these doses are given at a very low dose rate that protects critical organs.
When applying this technique for partial breast irradiation, the prescription dose will have to be calculated taking into account the biologic behavior of breast cancer cells $(14,15)$. The BED of a very low dose rate partial breast irradiation should be at least equivalent to the BED of a classical $50 \mathrm{~Gy}$ treatment in 25 fractions over 5 weeks.

Partial breast irradiation does decrease safety margins. This could be at the origin of a higher risk for local relapse as compared with external beam whole breast radiotherapy. Available follow-up data for partial breast irradiation are not yet long enough. We believe, however, that if performed using modern techniques and on carefully selected patients, excellent results can be obtained, with minimal patient discomfort or side effects. The technique described here potentially brings this patient comfort even a step further: a rapid and precise treatment, at a very low dose rate, delivering low doses to organs at risk, and also offering the possibility to deliver to the CTV a nearly ablative radiation dose (20). More and more methods are presently being developed for precise external beam treatment, but in an organ such as the breast, close to the heart and continuously moving with respiration, brachytherapy remains an interesting technique for improving the therapeutic index.

\section{REFERENCES}

1. Van Dongen JA, Voogd AC, Fentiman IS. Long-term results of a randomized trial comparing breast-conserving therapy with mastectomy: European Organization for Research and Treatment of Cancer 10801 trial. J Natl Cancer Inst 2000;92: 1143-1150.

2. Poortmans P, Bartelink H, Horiot JC. The influence of the boost technique on local control in breast conserving treatment in the EORTC "boost versus no boost" randomised trial. Radiother Oncol 2004;72:25-33.

3. Polgar C, Fodor J, Orosz Z, et al. Electron and high-dose-rate brachytherapy boost in the conservative treatment of stage I-II breast cancer: First results of the randomized Budapest boost trial. Strahlenther Onkol 2002;178:615-623.

4. Vicini FA, Arthur DW. Breast brachytherapy: North American experience. Semin Radiat Oncol 2005;15:108-115.

5. Polgar C, Strnad V, Major T. Brachytherapy for partial breast irradiation: The European experience. Semin Radiat Oncol 2005; $15: 116-122$.

6. Godinez J, Gombos EC. A permanent breast seed implant as partial breast radiation therapy for early stage patients: In regards to Keller et al. Int J Radiat Oncol Biol Phys 2006;64:1611.

7. McKean E, editor. The new Oxford American dictionary. 2nd ed (online version). Oxford: Oxford University Press; 2005.

8. ICRU Report No. 50: Prescribing, recording, and reporting photon beam therapy. Bethesda, MD: International Commission on Radiation Units and Measurements; 1978.

9. Van Limbergen E, Briot E, Drijkoningen M. The source-skin distance measuring bridge: A method to avoid radiation teleangiectasia in the skin after interstitial therapy for breast cancer. Int J Radiat Oncol Biol Phys 1990;18:1239-1244.

10. Orton CG, Ellis F. A simplification in the use of the NSD concept in practical radiotherapy. Br J Radiol 1973;46:529-537.

11. Orton CG, Webber BM. Time-dose factor (TDF) analysis of dose rate effects in permanent implant dosimetry. Int $J$ Radiat Oncol Biol Phys 1977;2:55-60.
12. Pignol JP, Keller B, Rakovitch E, et al. First report of a permanent breast 103Pd seed implant as adjuvant radiation treatment for early stage breast cancer. Int J Radiat Oncol Biol Phys 2006;64:176-181.

13. McRae D, Dodgers J, Dritschilo A. Dose-volume and complications in interstitial implants for breast carcinoma. Int $J$ Radiat Oncol Biol Phys 1987;13:525-529.

14. Dale RG. Radiobiological assessment of permanent implants using tumour repopulation factors in the linear-quadratic model. Br J Radiol 1989;62:241-244.

15. Guerrero M, Allen L. Analysis of a large number of clinical studies for breast cancer radiotherapy : Estimation of radiobiological parameters for treatment planning. Phys Med Biol 2003;48:3307-3326.

16. Pierquin B. Précis de curiethérapie, endocuriethérapie et plésiocuriethérapie. Paris: Masson et Cie; 1964. p. 241-246.

17. European Council directive 96/29/Euratom laying down basic safety standards for the protection of the health workers and the general public against the dangers arising from ionizing irradiation. 13 May 1996; http://europa.eu.int/comm/energy/ nuclear/radioprotection/doc/legislation/99829_en.pdf.

18. Keller B, Sankreacha R, Rakovitch E, et al. A permanent breast seed implant as partial breast radiation therapy for early stage patients: A comparison of Palladium-103 and Iodine-125 isotopes based on radiation safety considerations. Int J Radiat Oncol Biol Phys 2005;62:358-365.

19. Meinardi MT, van Veldhuisen DJ, Gietema JA. Prospective evaluation of early cardiac damage induced by epirubicin-containing adjuvant chemotherapy and locoregional radiotherapy in breast cancer patients. J Clin Oncol 2001;19:2746-2753.

20. Nickers P, Thissen B, Jansen N, et al. 192Ir or 125I prostate brachytherapy as a boost to external beam radiotherapy in locally advanced prostate cancer: A dosimetric point of view. Radiother Oncol 2006;78:47-52. 